\title{
Vibrio cholerae O1 Ogawa detoxified lipopolysaccharide structures as inducers of cytokines and oxidative species in macrophages
}

\author{
Correspondence \\ Ema Paulovičová \\ ema.paulovicova@savba.sk
}

Received 16 June 2009

Accepted 7 October 2009

\section{Ema Paulovičová, ${ }^{1}$ Elena Kováčová ${ }^{2}$ and Slavomír Bystrický ${ }^{1}$}

\author{
${ }^{1}$ Slovak Academy of Sciences, Institute of Chemistry, Centre for Glycomics, Centre of Excellence \\ GLYCOMED, Department of Immunochemistry of Glycoconjugates, Bratislava, Slovakia \\ ${ }^{2}$ Department of Animal Physiology and Ethology, Faculty of Natural Sciences, Comenius University, \\ Bratislava, Slovakia
}

\begin{abstract}
Multidrug resistance in several strains of Vibrio cholerae has encouraged anti-cholera vaccine developmental attempts using various subcellular moieties. In order to examine the immunological efficacy of detoxified LPS (dLPS)-derived saccharide immunogens, ex vivo activation of mouse peritoneal macrophages (MФs) was investigated. The immunomodulatory effect was evaluated via induction of the pro-inflammatory cytokines tumour necrosis factor- $\alpha$, interleukin (IL)- $1 \alpha$ and IL-6 and acceleration of nitric oxide (NO) and reactive oxygen species (ROS). Immunologically active structures triggered mouse peritoneal MФs to secrete cytokines and release NO/ROS, even at concentrations as low as $12.5 \mathrm{\mu g} \mathrm{m}^{-1}$. It was found that the $\mathrm{O}$-specific polysaccharide moiety was more immunologically efficient than the glycolipid one, probably due to the position of 3-deoxy-D-manno-octulosonic acid. The results revealed effective structure-immunomodulating relationships of dLPS-derived moieties that are desirable in subcellular anti-cholera vaccine design.
\end{abstract}

\section{INTRODUCTION}

Intestinal cholera infection is normally regarded as a noninvasive disease, because the causative agent, Vibrio cholerae, primarily affects the intestinal lumen. The existence of strains of $V$. cholerae resistant to several antibiotics and the increase in multidrug resistance have encouraged anti-cholera vaccine developmental attempts (Yamamoto et al., 2000; Roychowdhury et al., 2008). LPS, the major constituent of the outer membrane of Gramnegative bacteria, consists of a hydrophobic toxic lipid A, an outer and inner core oligosaccharide (core antigen or Rpolysaccharide) and a somatic antigen [O-specific polysaccharide (O-SP)]. These LPS domains represent the main areas of interest in cholera vaccine design (Chatterjee \& Chaudhuri, 2003, 2006; Erridge et al., 2002). A number of subcellular anti-cholera conjugate vaccines are currently being researched and/or are in pre-clinical development (Boutonnier et al., 2001; Chernyak et al., 2002; Gupta et al., 1992; Kossaczka et al., 2000).

Cholera infections frequently induce specific mucosal pathogen-associated immune responses. Monocyte-derived

Abbreviations: DeOAc-LPS, de-O-acetylated glycolipid; dLPS, detoxified LPS; EU, endotoxin unit; GM-CSF, granulocyte-macrophage colony stimulating factor; IFN, interferon; IL, interleukin; KDO, 3-deoxy-Dmanno-octulosonic acid; $\mathrm{M} \Phi$, macrophage; O-SP, O-specific polysaccharide; ROS, reactive oxygen species; TNF- $\alpha$, tumour necrosis factor- $\alpha$.
MФs represent the first line of host defence and are one of the most important cells acting in innate immune responses. Several groups of M $\Phi$ surface molecules are involved in specific interactions with LPS, particularly in the processes of cell recognition and activation (Chaby, 2004; Janeway \& Medzhitov, 1999; Alexander \& Rietschel, 2001), and induced secretion of pro-inflammatory cytokines has been documented (Scheibenbogen \& Andreesen, 1991; Haeffner-Cavaillon et al., 1988; Rabehi et al., 2001; Cavaillon, 1994). LPS-induced cell activation results in the generation of nitric oxide (NO) and reactive oxygen species (ROS). Both ROS and NO participate in host defence biological activities. It has been proposed that redox signalling plays an important role in the regulation of inflammatory responses (Forman \& Torres, 2002). Severe immunological inflammation or Gram-negative septic shock with exaggerated cytokine production could be manifested as a consequence of intact LPS overactivation of innate immunity. Pro-inflammatory cytokine overproduction is undesirable in cholera vaccine design. As lipid A exerts endotoxic activities, detoxification of LPS by lipid A elimination represents the first step in the preparation of a subcellular anti-cholera vaccine.

The aim of our study was to evaluate structureimmunomodulating relationships of novel $V$. cholerae detoxified LPS (dLPS)-derived moieties and the influence of different detoxification approaches on their effectiveness as immunomodulators. 


\section{METHODS}

Preparation of LPS. A dried acetone precipitate of $V$. cholerae $\mathrm{O} 1$ Ogawa El Tor was obtained from Dr Milan Seman (Vibrio Collection, Comenius University, Bratislava, Slovakia). LPS was extracted from the cells according to the method of Westphal \& Jann (1965).

Detoxification of LPS and preparation of the glycolipid fraction. For partial delipidation, LPS (100 mg) was dried over phosphate pentoxide and suspended in $10 \mathrm{ml}$ anhydrous hydrazine (Sigma-Aldrich Fine Chemicals) (Kondo et al., 1991). The solution was placed in a $37{ }^{\circ} \mathrm{C}$ water bath for $2 \mathrm{~h}$. Cold acetone was added slowly to $90 \%$ to precipitate the polysaccharide. Residual LPS was removed by ultracentrifugation. The final product was passed through a G-50 Sephadex column $(2.5 \times 50 \mathrm{~cm}$; Pharmacia $)$ in pyrogen-free water, sterile-filtered and freeze-dried. The de-Oacetylated glycolipid product, designated DeOAc-LPS, contained less than 10 endotoxin units (EU) $\mu \mathrm{g}^{-1}$ and $<1 \%$ protein or nucleic acid.

Preparation of core-attached $\mathbf{0}$-specific polysaccharide. The LPS was suspended in $5 \%$ acetic acid and heated at $100{ }^{\circ} \mathrm{C}$ for $2 \mathrm{~h}$. After cooling and $\mathrm{pH}$ neutralization, the mixture was ultracentrifuged at $64000 \mathrm{~g}$ for $5 \mathrm{~h}$ to remove lipid A. The supernatant was dialysed against water, passed through a Sephacryl (S-200) column, sterilefiltered $(0.2 \mu \mathrm{m})$ and freeze-dried. The product was designated O-SP.

Limulus amoebocyte lysate test. The Limulus amoebocyte lysate assay for endotoxin estimation was performed according to the technical information bulletin for the E-TOXATE kit (Sigma).

Animals. Male ICR mice aged 8-12 weeks were obtained from the breeding facility of the Institute of Experimental Pharmacology, Slovak Academy of Sciences (Dobrá Voda, Slovakia). Animal experiments were conducted in compliance with Good Laboratory Practice/Organisation for Economic Co-operation and Development guidelines, according to the ethical guidelines issued by the Institute of Virology, Slovak Academy of Sciences.

Preparation, culture and induction of MФs. To stimulate peritoneal M $\Phi$ proliferation, mice were injected intraperitoneally with $2 \mathrm{ml}$ sterile $5 \%$ thioglycolate broth (Difco Laboratories) (Park \& Rikihisha, 1991). Five days later, the mice were sacrificed by cervical dislocation, and $\mathrm{M} \Phi$ s were removed by peritoneal lavage using Hanks' balanced salt solution. The cells were washed by centrifugation, and $5 \times 10^{6}$ cells were resuspended in RPMI 1640 with L-glutamine supplemented with heat-inactivated fetal bovine serum and placed in each well of a 24-well tissue culture microplate (Sarstedt). Plates were incubated for $2 \mathrm{~h}$ at $37{ }^{\circ} \mathrm{C}$ in a humidified atmosphere of $5 \% \mathrm{CO}_{2}$. After the removal of non-adherent cells, at least $96 \%$ of the adherent cells, which were used in further experiments, were MФs according to morphological and phagocytic criteria (Akagawa \& Tokumaga, 1985). The medium was replaced with $0.5 \mathrm{ml}$ fresh medium containing LPSderived stimulants. After cultivation, the supernatants were collected and stored at $-70{ }^{\circ} \mathrm{C}$.

Immunophenotyping of peritoneal lavage MФs. For immunocytometric assays, cells were stained directly from peritoneal lavage (single or dual staining) and evaluated by flow cytometry using a FC 500 flow cytometer (Beckman Coulter) equipped with a $488 \mathrm{~nm}$ argon laser and a collinear $637 \mathrm{~nm}$ helium/neon laser, running under CXP software. For each sample, a histogram of $10^{4}$ fluorescent cells was generated and analysed. The following rat anti-mouse monoclonal antibodies were applied: fluorescein isothiocyanate-conjugated CD11b (Antigenix America) and phycoerythrin-conjugated CD14 (BD Pharmingen). The appropriate antibody isotype-negative controls (anti-IgG1 or anti-IgG2a) were used separately. The conjugated monoclonal antibodies $(5 \mu \mathrm{l})$ and peritoneal lavage samples $(50 \mu \mathrm{l})$ were added to $5 \mathrm{ml}$ sterile Falcon tubes (Becton Dickinson) and incubated for $30 \mathrm{~min}$ in the dark at $4{ }^{\circ} \mathrm{C}$. The lysis of erythrocytes was performed with $250 \mu \mathrm{l}$ OptiLyse C lysing solution (Immunotech). Lysis was stopped after $30 \mathrm{~min}$ of incubation in the dark at $4{ }^{\circ} \mathrm{C}$ by the addition of PBS ( $\mathrm{pH}$ 7.2) and the samples were evaluated by dualcolour flow cytometry.

Preparation of the polysaccharides. Solutions of the polysaccharide compounds O-SP and DeOAc-LPS were prepared at concentrations of $12.5,25$ and $50 \mu \mathrm{g} \mathrm{ml}^{-1}$ in complete RPMI 1640 and sterilized by filtration through a $0.22 \mu \mathrm{m}$ membrane (Millipore).

Determination of cytokines. The induction of tumour necrosis factor (TNF)- $\alpha$, interleukin (IL)- $1 \alpha$ and IL- 6 was measured in $\mathrm{M} \Phi$ cell culture supernatants after 3,6 and 24 h of cultivation with O-SP or DeOAc-LPS, using a flow cytometry fluorescent bead-based multiplex assay [Mouse Th1/Th2 10plex FlowCytomix Multiplex for quantitative detection of granulocyte-macrophage colony stimulating factor (GM-CSF), interferon (IFN)- $\gamma$, IL- $1 \alpha$, IL-2, IL-4, IL-5, IL-6, IL-10, IL17 and TNF- $\alpha$; Bender MedSystems]. Cytokine production of unstimulated cell populations was used to determine the baseline values.

Quantitative determination of ROS. M $\Phi$ cell culture supernatants that had been cultivated for 3, 6 and $24 \mathrm{~h}$ with O-SP or DeOAc-LPS were assayed for their total content of free radicals. A reagent kit based on the ability of chlorophyllin to transfer electrons due to its electron-rich double-bonds structure (Free radicals kit; Sevapharma) was used. The quantification of free radicals was determined using a calibration based on an $\mathrm{Fe}^{2+} / \mathrm{Fe}^{3+}$ reaction and was expressed as mmol $\mathrm{Fe}^{2+} 1^{-1}$. ROS production of unstimulated cell populations was used to determine the baseline value.

Quantitative determination of $\mathrm{NO}_{2}^{-}$production by MФs. This assay was carried out as described by Ding et al. (1988). Briefly, MФ cell culture supernatants collected after 3, 6 and $24 \mathrm{~h}$ of incubation with O-SP or DeOAc-LPS at different concentrations were used in the assay. A microtitre plate was used with standard $\mathrm{NaNO}_{2}$ solutions (serial dilutions from $0.25 \mathrm{mM}$ ) and equal volumes of the tested supernatants. Freshly prepared Griess reagent was added to each well and the $A_{550}$ was measured. $\mathrm{NO}_{2}^{-}$production of unstimulated cell populations was used to determine the baseline value.

Statistics. The results are presented as means \pm SEM. All data were statistically analysed by a one-way analysis of variance and a Bonferroni test to determine differences within the groups. $P$ values less than 0.05 were considered to be significant. Analyses were performed using OriginPro 7.5 software (OriginLab).

\section{RESULTS AND DISCUSSION}

Detoxification of LPS represents the first step in the creation of safe, lipid A-free, LPS-derived immunogenic derivatives. In our experiments, two detoxification procedures were applied, acid and base hydrolysis. Two potent immunogens, O-specific polysaccharide attached to the core polysaccharide (O-SP) and glycolipid (DeOAc-LPS), were prepared. The latent toxicity of both constructs was determined using a Limulus amoebocyte lysate test. Both

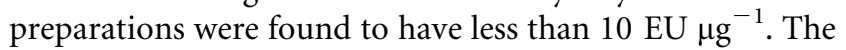
induction of pro-inflammatory cytokine secretion and $\mathrm{NO}$ and ROS generation was studied using mouse peritoneal MФs ex vivo. We observed a pattern of TNF- $\alpha$, IL- $1 \alpha$ and IL-6 dose-dependent stimulated release in murine intra- 
peritoneal MФs from mice pre- stimulated with thioglycolate broth. As a consequence of pre-stimulation, a 2.05-fold increase in the peritoneal lavage monocyte/M $\Phi$ population in comparison with unstimulated animals $(89.7 \% \pm 0.94$ $\mathrm{CD}_{11 b^{+}} \mathrm{CD} 4^{+}$cells vs $43.7 \pm 0.63 \%$ ) was detected by two-colour flow cytometry analysis. The actual morphology and cell counts were determined microscopically after staining with Türk's solution. The MФ cell monolayer was incubated in medium containing dLPS-derived immunogens. Spontaneous cytokine release from unstimulated cells was used as a negative control. The activation of adherent cells with the two immunogens resulted in the secretion of the pro-inflammatory cytokines TNF- $\alpha$, IL- $1 \alpha$ and IL-6. The other cytokines in the Th1/Th2 multiplex panel (GMCSF, IFN- $\gamma$, IL-2, IL-4, IL-5, IL-10 and IL-17) were negative.

To evaluate the effect of the dLPS-derived immunogens on pro-inflammatory cytokine secretion, the method was carried out using different concentrations of the immunogens (12.5-50 $\mathrm{g} \mathrm{ml}^{-1}$ ) (Figs 1 and 2). A concentration of $12.5 \mu \mathrm{g} \mathrm{ml}^{-1}$ was found to be sufficient for both substances to produce a significant induction of cytokine secretion $(P$ $<0.001)$.

Increasing concentrations of the polysaccharide and glycolipid moieties resulted in a dose-dependent increase in induced secretion of the pro-inflammatory cytokines IL6 and TNF- $\alpha$. In contrast, IL- $1 \alpha$ secretion appeared to be dose-independent, with the maximum stimulation being reached at $25 \mu \mathrm{g}$ immunogen $\mathrm{ml}^{-1}$. In this case, both immunogens exhibited a similar dose response. The extent of cytokine secretion varied with the given immunogen. When compared with DeOAc-LPS, O-SP induced 18.3-fold higher stimulated peak secretion of TNF- $\alpha$. Stimulation with O-SP also resulted in a 2.6 -fold peak increase in IL- $1 \alpha$ secretion and a 3.3-fold increase in IL-6 in comparison with DeOAc-LPS stimulation.

The time course of cytokine secretion in response to the stimuli was followed. The secretion of TNF- $\alpha$, IL- $1 \alpha$ and IL- 6 was measured at different time intervals $(3,6$ and $24 \mathrm{~h}$ ) after MФ stimulation (Figs 1 and 2). It was of interest that, although the extent of cytokine secretion in response to DeOAc-LPS was less than that induced by O-SP, the time course of secretion of the relevant cytokines was similar, with the exception of TNF- $\alpha$. DeOAc-LPS- and OSP-induced secretion of IL- $1 \alpha$ peaked after $3 \mathrm{~h}$, followed by a substantial decrease reaching low or undetectable values $24 \mathrm{~h}$ after stimulation. The time course of TNF- $\alpha$ secretion was clearly different, with the most significant increase being observed during the first $3 \mathrm{~h}$ in response to DeOAcLPS. The O-SP-stimulated peak amount of TNF- $\alpha$
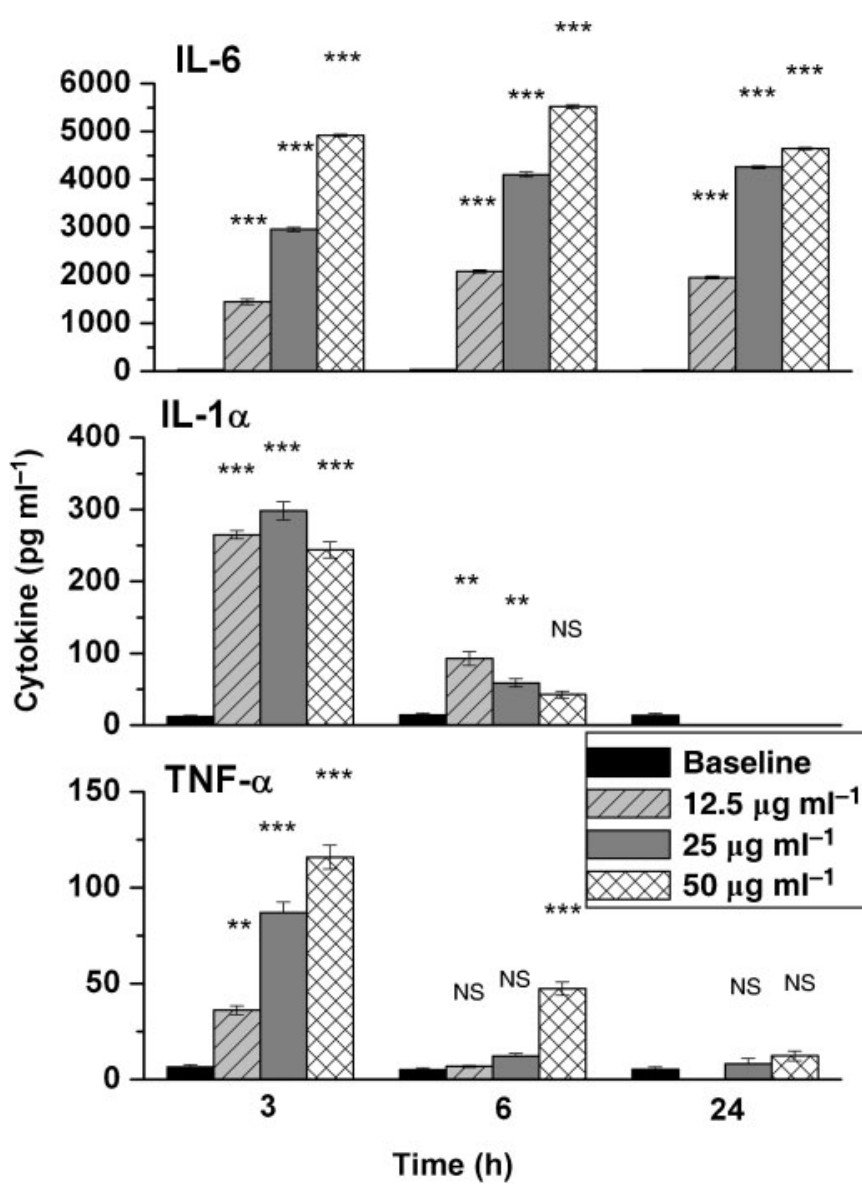

Fig. 1. Time-dependent cytokine secretion induced by the glycolipid moiety (DeOAcLPS) derived from LPS detoxified by base hydrolysis. Murine peritoneal MФs were stimulated for 3,6 or $24 \mathrm{~h}$ with $12.5-50 \mu \mathrm{g}$ DeOAc-LPS $\mathrm{ml}^{-1}$. Spontaneous (baseline) IL-6 and TNF- $\alpha$ secretion of unstimulated MФs was less than 22 and $15 \mathrm{pg} \mathrm{ml}^{-1}$, respectively. The data represent means \pm SEM of triplicate values. Comparisons of data groups were performed against unstimulated baseline cytokine secretion levels. Statistically significant differences: **, $P<0.01$; ${ }^{\star \star \star}, P<0.001$. NS, Not significant. 

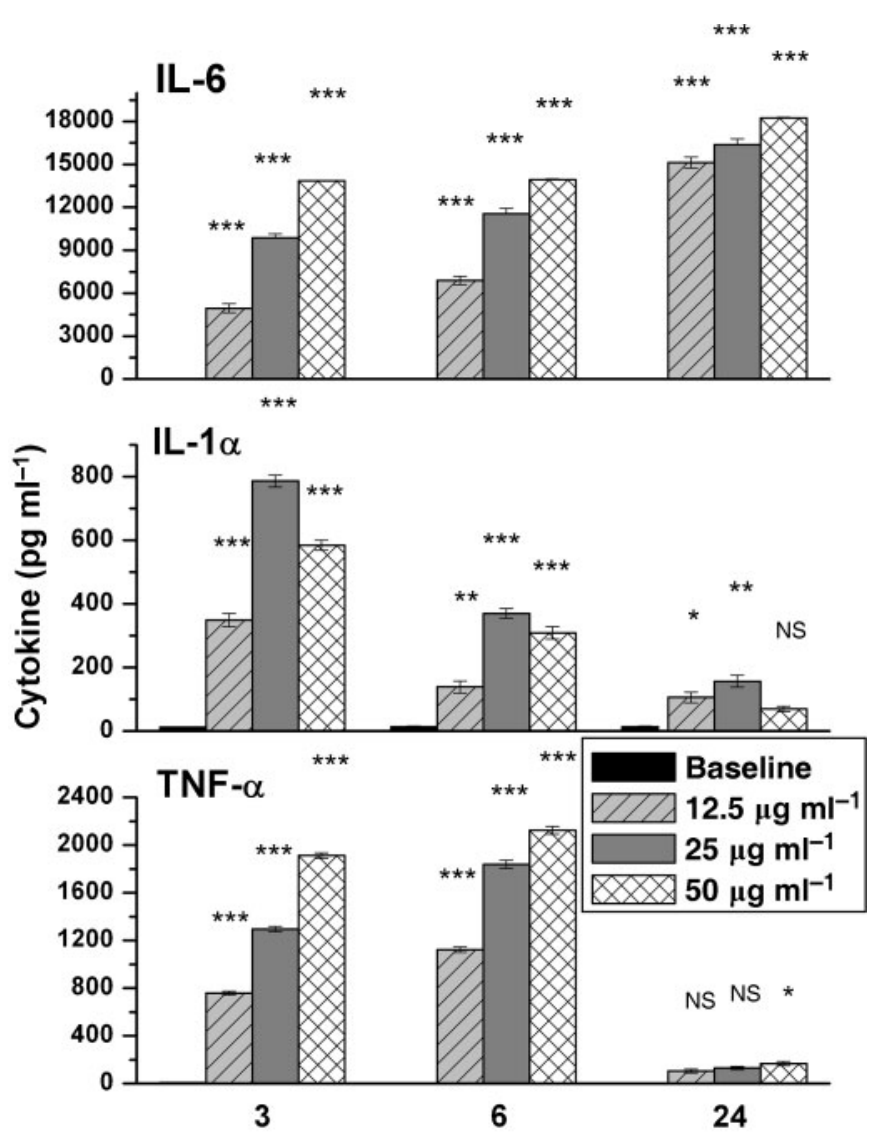

Time (h)
Fig. 2. Time-dependent cytokine pattern induced by O-SP derived from LPS detoxified by acid hydrolysis. Murine peritoneal MФs were stimulated for 3,6 or $24 \mathrm{~h}$ with 12.5$50 \mu \mathrm{g} \mathrm{O}-\mathrm{SP} \mathrm{ml}^{-1}$. Spontaneous unstimulated IL- 6 and TNF- $\alpha$ secretion was less than 22 and $15 \mathrm{pg} \mathrm{ml}^{-1}$, respectively. The data represent means \pm SEM of triplicate values. Comparisons of data groups were performed against unstimulated baseline cytokine secretion levels. Statistically significant differences: ${ }^{*}, P$ $<0.05 ;{ }^{* \star}, P<0.01$; ${ }^{\star \star *}, P<0.001$. NS, Not significant. culminated $6 \mathrm{~h}$ after M stimulation. Twenty-four hours after activation, the maximum peak levels of TNF- $\alpha$ were reduced by 89.1 and $90.8 \%$, respectively, compared with the levels observed $3 \mathrm{~h}$ after $M \Phi$ activation. DeOAc-LPSand O-SP-induced IL-6 levels exhibited a time-dependent increase and remained significantly elevated $(P<0.001)$ at $24 \mathrm{~h}$ after activation.

The time- and concentration-dependent ROS release was also determined after 3, 6 and $24 \mathrm{~h}$ of $\mathrm{M} \Phi$ activation (Fig. 3). DeOAc-LPS- and O-SP-stimulated production of ROS peaked after $3 \mathrm{~h}$, and then declined. The differences between ROS liberation by the two immunogens were observed throughout the concentration range. O-SP induced a 1.11-fold higher maximal peak value compared with DeOAc-LPS. Twenty-four hours after DeOAc-LPS and O-SP activation of peritoneal MФs, the maximum peak levels of ROS were reduced to 57.2 and $52.4 \%$, respectively, compared with the levels observed $3 \mathrm{~h}$ after M $\Phi$ activation. The maximal values reached were detected following stimulation with $50 \mu \mathrm{g}$ DeOAc-LPS or O-SP $\mathrm{ml}^{-1}$. The kinetics of the stable end metabolite $\mathrm{NO}_{2}^{-}$was measured to determine the induction of NO. The results of the dose- and activation time-dependent release of $\mathrm{NO}_{2}^{-}$ are summarized in Table 1.
The results of this study indicate the capability of these two dLPS-derived structures to induce pro-inflammatory mediators in MФs with respect to the extent of reduction or total elimination of the original toxicity of LPS. The important question was the impact of the two different chemical detoxification approaches on the immunomodulation capacity of the two derivatives.

LPS, a strong activator of innate immunity, is frequently associated with immunological inflammation or Gramnegative septic shock. Receptor-mediated attachment of LPS results in the activation of MФs followed by the release of inflammatory mediators, including various pro-inflammatory ILs such as IL- $1 \beta$, IL- $1 \alpha$, IL-6, IL- 8 and IL- 12 , IFNs such as IFN- $\gamma$, and chemokines and growth factors such as MCP-1, GC-CSF and TNF- $\alpha$. The cytokine triad of TNF- $\alpha$, IL- $1 \alpha$ and IL- 6 represents the main LPS-induced response. Our study of dLPS-derived substances showed the induction of only this triad (Figs 1 and 2), whilst the secretion of other cytokines and growth factors was not detected by FlowCytomix analysis. The close association between IL-1 and IL-6 levels and the severity of septic shock has been documented. Moreover, IL-1 markedly potentiates the toxic effect of TNF- $\alpha$ in animal experiments (Waage et al., 1991). TNF- $\alpha$ regulates cytokine and 


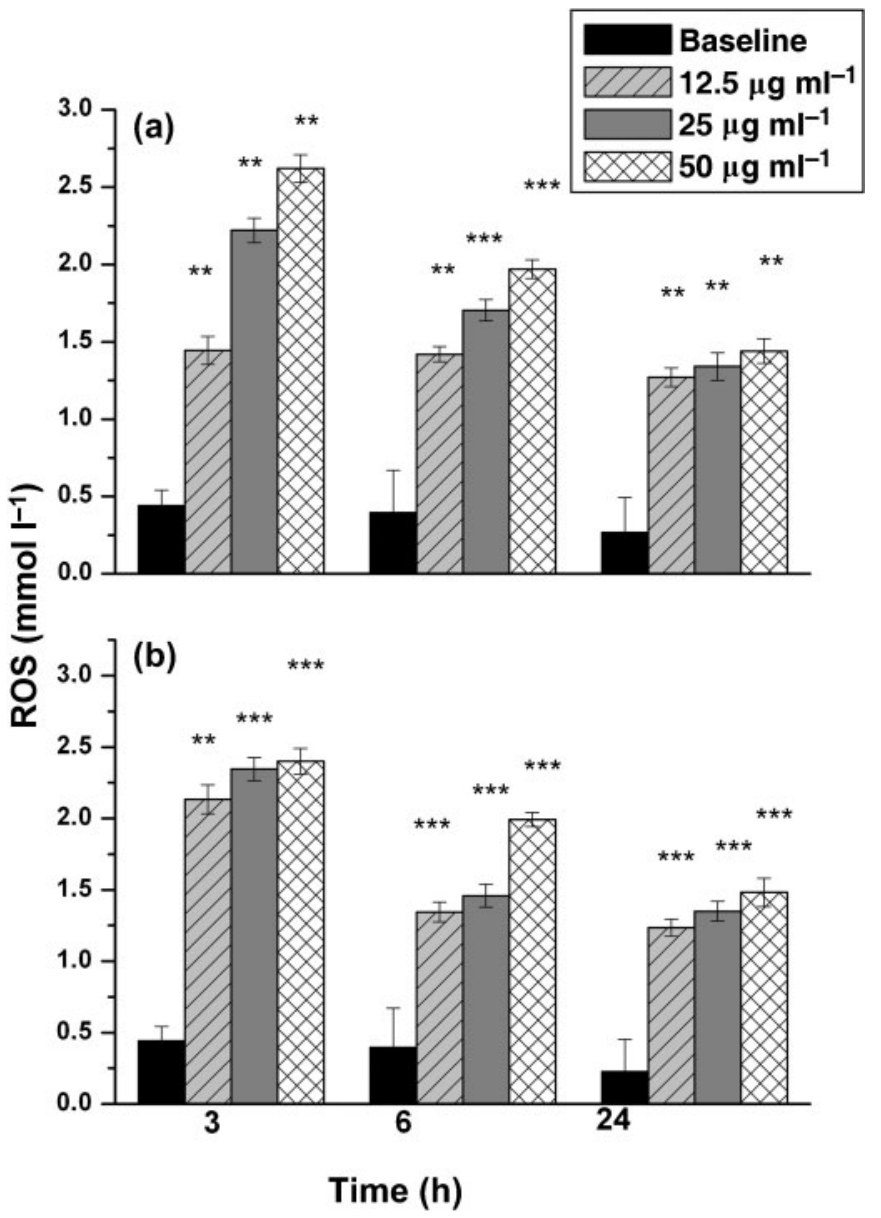

Fig. 3. Time- and concentration-dependent release of ROS induced by the LPS-derived saccharide moieties O-SP (a) and DeOAcLPS (b). The data represent means \pm SEM of triplicate values. Comparisons of data groups were performed against unstimulated baseline ROS expression levels using ANOVA. Statistically significant differences: ${ }^{* *}, P<0.01$; ${ }^{\star \star *}, P<0.001$. NS, Not significant. chemokine gene expression in a number of cells involved in the host response to infection and initiates a cascade of cytokines associated with infection. The effective biological activities of TNF- $\alpha$ comprise activation of $T$ and $B$ lymphocytes, MФs and natural killer cells; fever induction; acute-phase protein release; and endothelial cell activation. The primary source for TNF- $\alpha$ is activated monocytes and MФs, especially after priming by IFN- $\gamma$. It is a key regulator of other pro-inflammatory cytokines such as IL- $1 \alpha$, IL- 6 and IL-8. The generalized overproduction of TNF- $\alpha$ during Gram-negative bacterial infections causes widespread tissue damage and represents a common cause of circulatory

Table 1. $M \Phi$ release of $\mathrm{NO}_{2}^{-}\left(\mathrm{mmol} \mathrm{I}^{-1}\right)$ induced by DeOAcLPS and O-SP

\begin{tabular}{|c|c|c|c|c|c|c|}
\hline \multirow{2}{*}{$\begin{array}{l}\text { Concn of stimulant } \\
\left(\mu \mathrm{g} \mathrm{ml}^{-1}\right)\end{array}$} & \multicolumn{3}{|c|}{ DeOAc-LPS } & \multicolumn{3}{|c|}{ O-SP } \\
\hline & $3 \mathrm{~h}$ & $6 \mathrm{~h}$ & $24 \mathrm{~h}$ & $3 \mathrm{~h}$ & $6 \mathrm{~h}$ & $24 \mathrm{~h}$ \\
\hline 12.5 & 3.44 & 5.27 & 16.95 & 6.74 & 8.37 & 38.97 \\
\hline 25 & 17.83 & 18.26 & 40.23 & 3.44 & 7.81 & - \\
\hline 50 & 10.62 & 121.75 & 38.34 & 3.44 & 34.69 & 33.52 \\
\hline
\end{tabular}

shock leading to death. IL- $1 \alpha$ is a highly inflammatory cytokine similar to TNF- $\alpha$ in many aspects. It has a major influence on diverse cell populations and exerts biological effects including fever and increased synthesis of acutephase proteins. IL- $1 \alpha$ is secreted by MФs activated by a number of different stimuli such as TNF- $\alpha$ and/or bacterial endotoxin.

IL-6 has usually been regarded as a pro-inflammatory cytokine induced by LPS simultaneously with TNF- $\alpha$ and IL-1. IL-6 is frequently exploited as a marker of systemic activation of pro-inflammatory cytokines. Although IL-6 stimulates liver secretion of acute-phase proteins, it has anti-inflammatory properties as well. IL- 6 downregulates the synthesis of IL- 1 and TNF- $\alpha$. The ratio of IL- 6 to TNF$\alpha$ may be predictive with regard to chronic inflammation (Koj, 1985; Tilg et al., 1994; Opal \& dePalo, 2000; Dube et al., 2004).

Based on our results with dLPS-derived substances (Fig. 1 and Fig. 2), the various cytokine interactions could be supposed to include IL-6-promoted downregulation of IL$1 \alpha$ and TNF- $\alpha$. The generation of cytokines was more pronounced with O-SP stimulation, probably due to the structural arrangement of the molecule and epitope accessibility [probably located in the core 3-deoxy-D- 
manno-octulosonic acid (KDO) region, see below]. The production of ROS (Fig. 3) simultaneously with proinflammatory TNF- $\alpha$, IL- $1 \alpha$ and IL- 6 cytokine release demonstrated that effective $M \Phi$ immunoenhancement is more expressive with O-SP.

The reactive L-arginine-derived free radical $\mathrm{NO}$ is produced by NO synthase. NO is generated in $\mathrm{M} \Phi$ s following exposure to various stimuli, such as TNF- $\alpha$, IL- $1 \beta$, IFN- $\gamma$ and LPS (Chi et al., 2003). Cytokine- and dLPS-triggered production of $\mathrm{NO}_{2}^{-}$was observed with both LPS-derived moieties (Table 1). Excreted TNF- $\alpha$ and IL-1 might synergistically affect $M \Phi$ activity over the time course studied. $\mathrm{NO}_{2}^{-}$synthesis occurs at 3-6 h post-activation and progresses linearly up to $24 \mathrm{~h}$ (Mosser \& Zhang, 2008). Our results (Table 1) are in good agreement with these observations. Both tested substances induced an increase in $\mathrm{NO}_{2}^{-}$values in a time- and dose-dependent manner.

Based on these results, it was concluded that the observed pro-inflammatory cytokine trigger and ROS/NO generation corresponded to $\mathrm{M} \Phi$ activation by the O-SP and DeOAcLPS fractions of LPS. This observation will be important for future safe and efficient immunization/vaccination with subcellular vaccines based on LPS-derived fragments. Generally, the immunomodulative properties of LPS are ascribed to the polysaccharide core antigenic moieties and the somatic O-antigen. The inner-core-derived oligosaccharides trigger mononuclear cells to produce the immunostimulating cytokine IL-1 (Lebbar et al., 1986). Synthetic or natural oligosaccharides such as the core-derived heptose (Hep) II-Hep I-KDO I trisaccharide induce the release of IL-1 from human mononuclear cells (Johnson, 1994). The minimal structure required to trigger IL-1 secretion seems to be a disaccharide that contains a neutral sugar (e.g. heptose) (Rietschel et al., 1996).

As predominant surface structures, $\mathrm{O}$-antigens are highly immunogenic and $\mathrm{O}$-specific antibodies are frequently used for serotyping, in clinical diagnosis, and for epidemiology and taxonomy. Moreover, O-antigens are involved in both the activation and inhibition of the complement cascade (Tanamoto et al., 1984; Viswanathan et al., 2004). The O-polysaccharide has also been associated with the cell attachment of LPS in host-parasite interactions and the induction of cytokine release.

Various reported data have indicated that the polysaccharide moiety of LPS molecules is important for its biological activity and is involved in stimulation of human monocytes (Otterlei et al., 1993; Cavaillon et al., 1989). KDO, which forms the link between lipid A and the polysaccharide in LPS, is a remarkably immunopotent molecule, as illustrated by LPS mutants lacking the entire polysaccharide chain except for KDO (Cavaillon, 1994). Pro-inflammatory cytokine release especially IL-1 by monocytes as a response to KDO has been documented (Lebbar et al., 1986).

Our results on $\mathrm{M} \Phi$ cytokine induction with detoxified LPS-derived immunogenic structures are in agreement with the important role of the core $\mathrm{KDO}$ region in the immunomodulation process. Immunogenic O-SP isolated by acid hydrolysis of $V$. cholerae LPS has the KDO structure readily accessible in a terminal position. In contrast, the KDO structure in the second immunogen that we examined, DeOAc-LPS, is internal. The induction of proinflammatory cytokines and $\mathrm{ROS} / \mathrm{NO}_{2}^{-}$, which was more profound in the case of the O-SP moiety, may be the result of such a structural arrangement.

Novel trends in the development of an anti-cholera vaccine are directed towards dLPS-derived structures and their ability to induce a potent immunogenic effect. Subcellular vaccines represent one of the recent trends in saccharide vaccine design. Conjugation of thymus-independent saccharide moieties with a protein carrier shifts such constructs towards thymus dependency. It has been shown to be possible to make an effective immunogen based on subcellular low-molecular-mass oligosaccharides (Chernyak et al., 2002; Paulovičová et al., 2006). A better understanding of the structure-immunomodulating activity relationship will permit the development of selective anti-cholera subcellular vaccines.

The present investigation of dLPS-derived structures revealed the effective acceleration of $\mathrm{ROS} / \mathrm{NO}_{2}^{-}$production and pro-inflammatory TNF- $\alpha$, IL- $1 \alpha$ and IL- 6 cytokine release by stimulated mouse peritoneal MФs. These results indicate an effective immunomodulating activity of two $V$. cholerae O1 Ogawa El Tor dLPS-derived structures and provide encouraging findings for the use of such derivatives containing the core KDO region in subcellular cholera vaccine development.

\section{ACKNOWLEDGEMENTS}

This work was supported by the Slovak Research and Development Agency under contract no. APVV-0032-06 and by the Grant Agency of Slovak Academy of Sciences VEGA no. 2/7029/27.

\section{REFERENCES}

Akagawa, K. S. \& Tokumaga, T. (1985). Lack of binding of bacterial lipopolysaccharide to mouse lung macrophages and restoration of binding by interferon gamma. J Exp Med 162, 1444-1459.

Alexander, Ch. \& Rietschel, E. T. (2001). Bacterial lipopolysaccharides and innate immunity. J Endotoxin Res 7, 167-202.

Boutonnier, A., Villeneuve, S., Nato, F., Dassy, B. \& Fournier, J. M. (2001). Preparation, immunogenicity, and protective efficacy, in a murine model, of a conjugate vaccine composed of the polysaccharide moiety of the lipopolysaccharide of Vibrio cholerae O139 bound to tetanus toxoid. Infect Immun 69, 3488-3493.

Cavaillon, J. M. (1994). Cytokines and macrophages. Biomed Pharmacother 48, 445-453.

Cavaillon, J. M., Fitting, C., Caroff, M., Haeffner, C. A. \& Vaillon, N. (1989). Dissociation of cell-associated interleukin-1 (IL-1) and IL-1 release induced by lipopolysaccharide and lipid A. Infect Immun 57, 791-797. 
Chaby, R. (2004). Lipopolysaccharide-binding molecules: transporters, blockers and sensors. Cell Mol Life Sci 61, 1697-1713.

Chatterjee, S. N. \& Chaudhuri, K. (2003). Lipopolysaccharides of Vibrio cholerae. I. Physical and chemical characterization. Biochim Biophys Acta 1639, 65-79.

Chatterjee, S. N. \& Chaudhuri, K. (2006). Lipopolysaccharides of Vibrio cholerae. III. Biological functions. Biochim Biophys Acta 1762, $1-16$.

Chernyak, A., Kondo, S., Wade, T. K., Meeks, M. D., Alzari, P. M., Fournier, J. M., Taylor, R. K., Kovac, P. \& Wade, W. F. (2002). Induction of protective immunity by synthetic Vibrio cholerae hexasaccharide derived from Vibrio cholerae O1 Ogawa lipopolysaccharide bound to a carrier protein. J Infect Dis 185, 950-952.

Chi, D. S., Qui, M., Krishnaswamy, G., Li, C. H. \& Stone, W. (2003). Regulation of nitric oxide production from macrophages by lipopolysaccharide and catecholamines. Nitric Oxide 8, 127-132.

Ding, A. H., Nathan, C. F. \& Stuehr, D. J. (1988). Release of reactive nitrogen intermediate and reactive oxygen intermediates from mouse peritoneal macrophages. Comparison of activating cytokines and evidence for independent production. J Immunol 141, 2407-2412.

Dube, P. H., Handley, S. A., Lewis, J. \& Miller, V. L. (2004). Prospective role of interleukin-6 during Yersinia enterocolitica infection is mediated through the modulation of inflammatory cytokines. Infect Immun 72, 3561-3570.

Erridge, C., Bennett-Guerrero, E. \& Poxton, I. R. (2002). Structure and function of lipopolysaccharides. Microbes Infect 4, 837-851.

Forman, H. J. \& Torres, M. (2002). Reactive oxygen species and cell signaling. Respiratory burst in macrophage signaling. Am J Respir Crit Care Med 166, S4-S8.

Gupta, R. K., Szu, S. C., Finkelstein, R. A. \& Robbins, J. B. (1992). Synthesis, characterization, and some immunological properties of conjugates composed of the detoxified lipopolysaccharide of Vibrio cholerae O1 serotype Inaba bound to cholera toxin. Infect Immun 60, 3201-3208.

Haeffner-Cavaillon, N., Bacle, F., Caroff, M. \& Cavaillon, J. M. (1988). Characteristics of lipopolysaccharide-induced interleukin-1 production by human monocytes. Clinical relevance in patients undergoing hemodialysis. Prog Clin Biol Res 272, 89-101.

Janeway, C. A., Jr \& Medzhitov, R. (1999). Innate immunity: lipoproteins take their Toll on the host. Curr Biol 9, R879-R882.

Johnson, A. G. (1994). Molecular adjuvants and immunomodulators: new approaches to immunization. Clin Microbiol Rev 7, 277-289.

Koj, A. (1985). Biological functions of acute phase proteins. In The Acute Phase Response to Injury and Infection, pp. 139-144. Edited by A. H. Gordon \& A. Koj. Amsterdam: Elsevier.

Kondo, S., Zahringer, U., Seydel, U., Sinnwell, V., Hisatsune, K. \& Rietschel, E. T. (1991). Chemical structure of the carbohydrate backbone of Vibrio parahaemolyticus serotype 012 lipopolysaccharide. Eur J Biochem 200, 689-698.

Kossaczka, Z., Shiloach, J., Johnson, V., Taylor, D. N., Finkelstein, R. A., Robbins, J. B. \& Szu, S. C. (2000). Vibrio cholerae O139 conjugate vaccines: synthesis and immunogenicity of Vibrio cholerae O139 capsular polysaccharide conjugates with recombinant diphtheria toxin mutant in mice. Infect Immun 68, 5037-5043.

Lebbar, S., Cavaillon, J. M., Caroff, M., Ledur, A., Brade, H., Sarfati, R. \& Haeffner-Cavaillon, N. (1986). Molecular requirement for interleukin 1 induction by lipopolysaccharide-stimulated human mono- cytes: involvement of heptosyl-2-keto-deoxyoctulosonate region. Eur J Immunol 16, 87-91.

Mosser, D. M. \& Zhang, X. (2008). Activation of murine macrophages Current Protoc Immunol 83, 14.2.1-14.2.8.

Opal, S. M. \& dePalo, V. A. (2000). Anti-inflammatory cytokines. Chest 117, 1162-1172.

Otterlei, M., Sundan, A., Skjak-Braek, G., Ryan, L., Smidsrod, O. \& Espevik, T. (1993). Similar mechanisms of action of defined polysaccharides and lipopolysaccharides: characterization of binding and tumor necrosis factor alpha induction. Infect Immun 61, 19171925.

Park, J. \& Rikihisha, Y. (1991). Inhibition of Ehrlichia risticii infection in murine peritoneal macrophages by gamma interferon, a calcium ionophore and concanavalin A. Infect Immun 59, 3418-3423.

Paulovičová, E., Machová, E., Hoštacká, A. \& Bystrický, S. (2006). Immunological properties of complex conjugates based on Vibrio cholerae O1 Ogawa lipopolysaccharide antigen. Clin Exp Immunol 144, 521-527.

Rabehi, L., Irinopoulou, T., Cholley, N., Haeffner-Cavaillon, N. \& Carreno, M. P. (2001). Gram-positive and Gram-negative bacteria do not trigger monocytic cytokine production through similar intracellular pathways. Infect Immun 69, 4590-4599.

Rietschel, E. T., Brade, H., Holst, O., Brade, L., Muller-Leonnies, S., Mamat, U., Zahringer, U., Beckman, F., Seydel, U. \& other authors (1996). Bacterial endotoxin: chemical constitution, biological recognition, host response, and immunological detoxification. Curr Top Microbiol Immunol 216, 39-81.

Roychowdhury, A., Pan, A., Dutta, D., Mukhopadhyay, A. K., Ramamurthy, T., Nandy, R. K. \& Bhattacharya, S. K. (2008). Emergence of tetracycline-resistent Vibrio cholerae O1 serotype Inaba in Kolkata, India. Jpn J Infect Dis 61, 128-129.

Scheibenbogen, C. \& Andreesen, R. (1991). Developmental regulation of the cytokine repertoire in human macrophages: IL-1, IL-6, TNF- $\alpha$ and M-CSF. J Leukoc Biol 50, 35-42.

Tanamoto, K., Zähringer, U., McKenzie, G. R., Galanos, C., Rietschel, E. T., Lüderitz, O., Kusumoto, S. \& Shiba, T. (1984). Biological activities of synthetic lipid A analogs: pyrogenicity, lethal toxicity, anticomplement activity, and induction of gelation of Limulus amoebocyte lysate. Infect Immun 44, 421-426.

Tilg, H., Trehu, E., Atkins, M. B., Dinarello, C. A. \& Mier, J. W. (1994). Interleukin-6 (IL-6) as an anti-inflammatory cytokine: induction of circulating IL-1 receptor anagonist and soluble tumor necrosis factor receptor p55. Blood 83, 113-118.

Viswanathan, V. K., Sharma, R. \& Hecht, G. (2004). Microbes and their products - physiological effects upon mammalian mucosa. $A d v$ Drug Deliv Rev 56, 727-762.

Waage, A., Brandtzaeg, P., Espevik, T. \& Halstensen, A. (1991). Current understanding of the pathogenesis of Gram-negative shock. Infect Dis Clin North Am 5, 781-791.

Westphal, O. \& Jann, K. (1965). Bacterial lipopolysaccharides. Extraction with phenol-water and further application of the procedure. Methods Carbohydr Res 5, 83-91.

Yamamoto, T., Taneike, I., Tamura, Y. \& Wakisaka-Saito, N. (2000). Multiple drug resistance of Vibrio cholerae $\mathrm{O} 1$ and $\mathrm{O} 139$ isolated from various regions of the world: changes in the past 10 years. Acta Med Biol 48, 75-88. 